\title{
Investigating hydration dependence of dynamics of confined water: Monolayer, hydration water and Maxwell-Wagner processes
}

\author{
Johan Sjöström, ${ }^{1, a)}$ Jan Swenson, ${ }^{1}$ Rikard Bergman, ${ }^{1}$ and Shigeharu Kittaka ${ }^{2}$ \\ ${ }^{1}$ Department of Applied Physics, Chalmers University of Technology, SE-41296 Göteborg, Sweden \\ ${ }^{2}$ Department of Chemistry, Faculty of Science, Okayama University of Science, 1-1 Ridaicho, \\ Okayama 700-0005, Japan
}

(Received 18 October 2007; accepted 4 March 2008; published online 15 April 2008)

\begin{abstract}
The dynamics of water confined in silica matrices MCM-41 C10 and C18, with pore diameter of 21 and $36 \AA$, respectively, is examined by broadband dielectric spectroscopy $\left(10^{-2}-10^{9} \mathrm{~Hz}\right)$ and differential scanning calorimetry for a wide temperature interval (110-340 K). The dynamics from capillary condensed hydration water and surface monolayer of water are separated in the analysis. Contrary to previous reports, the rotational dynamics are shown to be virtually independent on the hydration level and pore size. Moreover, a third process, also reported for other systems, and exhibiting a saddlelike temperature dependence is investigated. We argue that this process is due to a Maxwell-Wagner process and not to strongly bound surface water as previously suggested in the literature. The dynamics of this process is strongly dependent on the amount of hydration water in the pores. The anomalous temperature dependence can then easily be explained by a loss of hydration water at high temperatures in contradiction to previous explanations. (C) 2008 American Institute of Physics. [DOI: 10.1063/1.2902283]
\end{abstract}

\section{INTRODUCTION}

Confined supercooled water has drawn a lot of attention in the last decade both for its relevance to fundamental physics, biology, and geology and as a possibility to have a glimpse into the glassy dynamics of water in the so called no mans land $(150-235 \mathrm{~K})$ where bulk water immediately crystallize. ${ }^{1}$ Close confinement hinders ice formation but consequently the supercooled liquid suffers from constraints induced by the confining system.

The viscosity (or the directly related cooperative alpha relaxation time) of a glass forming liquid generally follows a Vogel-Fulcher-Tamman (VFT) equation, $\quad \eta \propto \tau$ $=\tau_{0} \exp \left[D T_{0} /\left(T-T_{0}\right)\right]$. For high $D$ values this expression resembles an Arrhenius expression [Eq. (1)] and the liquid is said to be strong. A small $D$ implies a more rapid slowing down of the dynamics close to the glass transition and the liquid is labeled fragile. At high temperatures bulk water appears to be an altogether fragile liquid but for low temperatures, on the other side of the no mans land, it resembles a very strong one. To resolve this dual picture a fragile-tostrong transition (FST) of the viscosity (or the directly related cooperative $\alpha$ relaxation) was suggested to occur somewhere between 150 and $235 \mathrm{~K}^{2}$. During the last 3 years, quasielastic neutron scattering (QENS) and nuclear magnetic resonance (NMR) experiments have shown a very pronounced cross over from a fragile to a seemingly strong behavior of water in MCM41, ${ }^{3-5}$ quasi-two-dimensional confinement, ${ }^{6}$ and biomaterials ${ }^{7}$ at $220-225 \mathrm{~K}$ for ambient pressure. These findings are also supported by MD simulations. ${ }^{8}$ How to interpret this transition is, however, a subject of debate. ${ }^{9-11}$

\footnotetext{
${ }^{a)}$ Electronic mail: johan.sjostrom@chalmers.se.
}

The low temperature main dielectric relaxation of confined water (regardless the type of confinement) does not show fragile VFT behavior ${ }^{12}$ but follows the Arrhenius law

$$
\tau=\tau_{0} \exp \frac{E_{a}}{k_{B} T}
$$

where $\tau_{0}$ describes the high temperature asymptote of the relaxation time, and $E_{a}$ is the activation energy, i.e., the energy barrier that the molecules must overcome in order to rotate. This process has been shown to exhibit typical behavior of a local relaxation ${ }^{12,13}$ and different possible origins have been discussed in several publications. ${ }^{14-16}$ Recently, it has been attributed to the so-called Johari-Goldstein relaxation. ${ }^{16}$ Unlike a cooperative $\alpha$ relaxation a local relaxation is not related to the viscosity which is required in order to determine the fragility. It is possible that the restricted geometry is too severe for extended cooperative motion to take place.

Among the different porous silica matrices the nanoporous silica mobil catalytic material No. 41 (MCM-41) have the most homogeneous and well defined pore size distribution in the range of $1-10 \mathrm{~nm} .{ }^{17}$ Dielectric studies of water confined in MCM-41 materials ${ }^{18-20}$ and alike ${ }^{21-23}$ have been published. The spectra from these studies show the dynamical contribution of water superimposed on a large polarization dispersion, probably due to electrode polarization. In the only previous study of the hydration dependence of the dynamics the motions of hydroxyl groups and amorphous water have been separated and reported to show a strong hydration level $(\mathrm{H})$ dependence. ${ }^{19}$ With increasing $\mathrm{H}$ the water relaxations had an increasing activation energy. Simultaneously, the $\mathrm{OH}$ groups showed a drastic decrease of the high temperature asymptote of the relaxation time. A third 
process, attributed to the matrix, was also detected buried in the polarization dispersion and thus impossible to quantitatively analyze. ${ }^{19}$

A striking feature of nonmonotonic temperature dependence of the dynamics of water confined in nanoporous glasses with very large pores, ${ }^{24}$ very small soldalite cages of faujasite $^{25}$ and porous silica (SBA15) with larger pore sizes than our present system ${ }^{22}$ has previously been reported. The dynamics of the confined water in these cases became faster with increasing temperature but reached a maximum at 270-310 K. Thereafter the dynamics rapidly became much slower with increasing temperature. A model to explain this feature was introduced by Ryabov et $a{ }^{23}{ }^{23}$ It states that the change in the dynamics is due to a competition between the rotational activation energy and free volume related to the density of defects in the $\mathrm{H}$ bonded network. For high temperatures the free volume of the water molecules decreases drastically due to less defect density. The total relaxation time was then introduced as

$$
\tau=\tau_{0} \exp \left[\frac{E_{a}}{k_{B} T}+C \exp \left(\frac{-E_{d}}{k_{b} T}\right)\right],
$$

where $E_{d}$ is the defect formation energy and $C$ is a constant explained in Ref. 23. The model fails to explain why bulk network glass formers does not show similar behavior.

In this study we show that the dielectric relaxation of amorphous hydration and monolayer water show no dependence on hydration level. We follow the local dynamics of hydration water to temperatures above the FST proposed by neutron scattering and NMR, and show that dielectric relaxation does not participate in any such kinetic transition. Furthermore, by introducing a thin layer of Teflon between the sample and the upper electrode, the polarization contribution is suppressed and a slow process, following the previously mentioned saddlelike temperature dependence is detected. This process is highly dependent on the hydration level and we give a new alternative explanation for its origin.

\section{SAMPLE PREPARATION AND EXPERIMENT}

The nanoporous silica of the type MCM-41 was prepared by the modified Beck method. ${ }^{26}$ Long-chain alkyltrimethylammonium bromides $\mathrm{C}_{n} \mathrm{H}_{2 n+1}-\left(\mathrm{CH}_{3}\right)_{3} \mathrm{~N}^{+} \mathrm{Br}$ were used as the template organic reagent. The pore diameter is determined by the number of carbon atoms $n$ in the long alkyl group. The samples used in this work had $n=10$ and 18, subsequently called C10 (21 $\AA$ ) and C18 (36 ̊). The pores are ordered in a hexagonal structure but the actual pore walls are amorphous and thereby suitable for avoiding crystallization of the confined water. The inner walls are covered with hydroxyl groups. Thermal gravimetric adsorption measurements show ${ }^{27}$ that the pores are stable against drying and rehydration several times. Note that the size of the pores allow not more than six water molecules and $\mathrm{OH}$ groups on the diagonal of the $\mathrm{C} 10$ pores and a maximum of ten molecules in the C18 sample.

The material has been extensively investigated using neutron scattering, ${ }^{28,29}$ x-ray scattering ${ }^{30}$ and by DSC, Fourier transformed infrared spectroscopy (FTIR) spectroscopy, and gravimetrical adsorption measurements. ${ }^{27}$ The DSC measurements of Ref. 27 show that the water in C10 $(d$ $=21 \AA$ ) remains amorphous whereas substantial ice formation takes place for fully hydrated C18 $(d=36 \AA)$ at $240 \mathrm{~K}$. FTIR measurements reveals that the monolayer next to the pore walls in the C18 samples does not participate in the crystallization but remains amorphous.

The samples were hydrated by exposing them to $100 \%$ humidity at room temperature after being evacuated at $573 \mathrm{~K}$ for $24 \mathrm{~h}$ and left in vacuum until oven temperature decreased to room temperature. This treatment removes all the water from the pores and most of the surface hydroxyls.

The hydration levels mentioned in this paper is defined as $\mathrm{H}=$ (increase in mass during hydration)/(mass of sample directly from vacuum oven) and is thus determined on the basis of the "dry" sample. The reference to dry sample means sample measured within minute of exposure to atmospheric conditions. However, water contaminates the surfaces almost instantaneously so the $\mathrm{H}$ level is not a perfect quantitative amount. It should be regarded as an indication of the hydration level and points out trends in the hydration dependent properties. Some samples were prepared in an atmosphere of Ar. These had not been in contact to atmospheric conditions or water before measurements and are referred to as "inert samples."

The DSC experiments were performed on a TA Instruments DSC Q1000 using hermetically sealed aluminum pans. Experiments were performed using both controlled cryostat cooling and hyperquenching (lowering sample into liquid nitrogen before data was collected in the precooled cryostat).

The broadband dielectric measurements were performed using two different analyzers for the frequency ranges of $10^{-2}-3 \times 10^{6} \mathrm{~Hz}$ (Novocontrol, Alpha) and $10^{6}-10^{9} \mathrm{~Hz}$ (HP, 4192). The sample was placed in between two electrodes of 40 and $10 \mathrm{~mm}$ diameter for the broadband and high frequency analyzer, respectively. The temperature was within $0.3 \mathrm{~K}$ of the set point during all frequency scans.

In order to suppress polarization and conductivity effects a thin sheet $(100 \mu \mathrm{m})$ of Teflon was introduced for some of the samples between the sample and the top electrode. This made it possible to observe and analyze also a slow relaxation processes at relatively high temperatures (around $300 \mathrm{~K}$ ), without significantly affecting the low temperature processes that could be observed also without using the Teflon sheet.

\section{RESULTS}

\section{A. Calorimetric measurements}

The relative ice formation in the two pores is shown as a function of hydration level in Fig. 1 using estimations explained in the figure caption. The data show no sign of a glass transition for any sample. The inset shows typical calorimetric scans. For the $\mathrm{C} 10$ pores, with $\mathrm{H}=105 \%$ a negligible melting occurs at $0{ }^{\circ} \mathrm{C}$ corresponding to less than $4 \%$ of the confined water. This is attributed to surface water outside the 


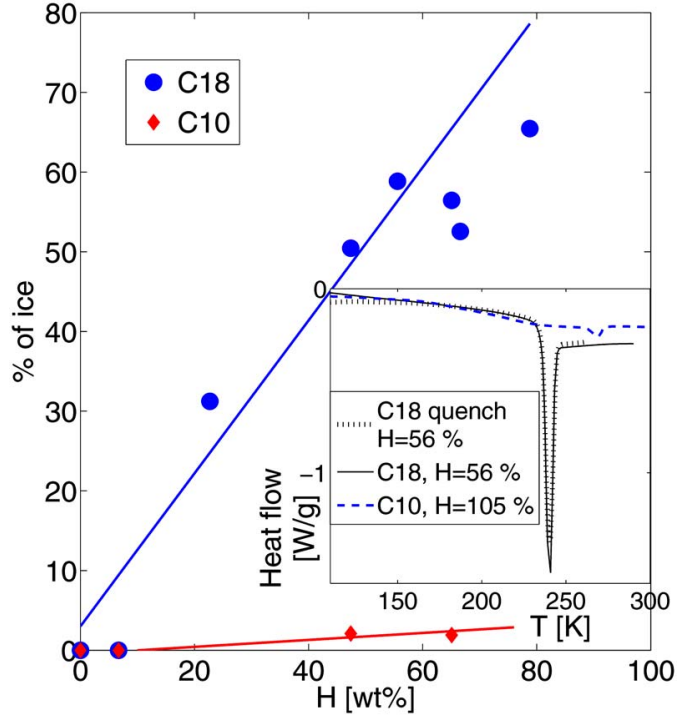

FIG. 1. (Color online) The percentage of crystallization of absorbed water as a function of hydration level. The percentage is calculated using the estimation (Ref. 31) that the heat of fusion is reduced by approximately $40 \%$ at $240 \mathrm{~K}$ and correcting for the mass of the hydroxyl groups. The solid lines are guides to the eye. The inset shows the DSC scans of samples cooled by $10 \mathrm{~K} / \mathrm{min}$ as well as by hyperquenching. The small melting feature at $0{ }^{\circ} \mathrm{C}$ for the $\mathrm{C} 10$ sample is attributed to surface water.

pores (see Sec. IV A). No other melting is detected which implies that all the water in the pores are amorphous. We also show scans of $\mathrm{C} 18$, one using hyperquenching and one using ordinary cooling by the cryostat. The ice formation corresponds to 56\% (quenching) and 59\% (cryostat cooling), respectively.

\section{B. Dielectric measurements}

The complex permittivity, $\epsilon^{*}(\omega)=\epsilon^{\prime}(\omega)-i \epsilon^{\prime \prime}(\omega)$, where $\epsilon^{\prime}$ is the dielectric constant and $\epsilon^{\prime \prime}$ the dielectric loss (Fig. 2), was measured for all samples. The complex part of the spectra were fitted using several Havriliak-Negami $(\mathrm{H}-\mathrm{N})$ functions and a conductivity term

$$
\epsilon^{*}=\epsilon^{\prime}-i \epsilon^{\prime \prime}=-i \frac{\sigma_{0}}{\epsilon_{0} \omega^{\delta}}+\epsilon_{\infty}+\sum_{i=1}^{N} \frac{\Delta \epsilon}{\left[1+(i \omega \tau)^{\alpha}\right]^{\beta}},
$$

where $\sigma_{0}$ is the de conductivity and $\epsilon_{0}$ the vacuum permittivity. $\epsilon_{\infty}$ is the high frequency asymptote of the permittivity, $\Delta \epsilon=\epsilon_{\text {stat }}-\epsilon_{\infty}$ is the "dielectric strength" $\left(\epsilon_{\text {stat }}\right.$ is the static field permitivity), $\tau$ is the characteristic relaxation time, $\alpha$ is the symmetric broadening of the process describing the deviation from Debye relaxation behavior ${ }^{32}$ and the $\beta$ parameter controls the asymmetric broadening (on the high-frequency side). For $\beta=1$ the process is symmetric (on a log scale of frequency) and known as the Cole-Cole (C-C) function. The $\tau$ parameter then equals the inverse of the peak angular frequency $\omega_{p}^{-1}$ of $\epsilon^{\prime \prime}$.

The inert samples did not show any relaxation processes within our experimental limits. This means that if any small residues of hydroxyl groups exist they do not relax via
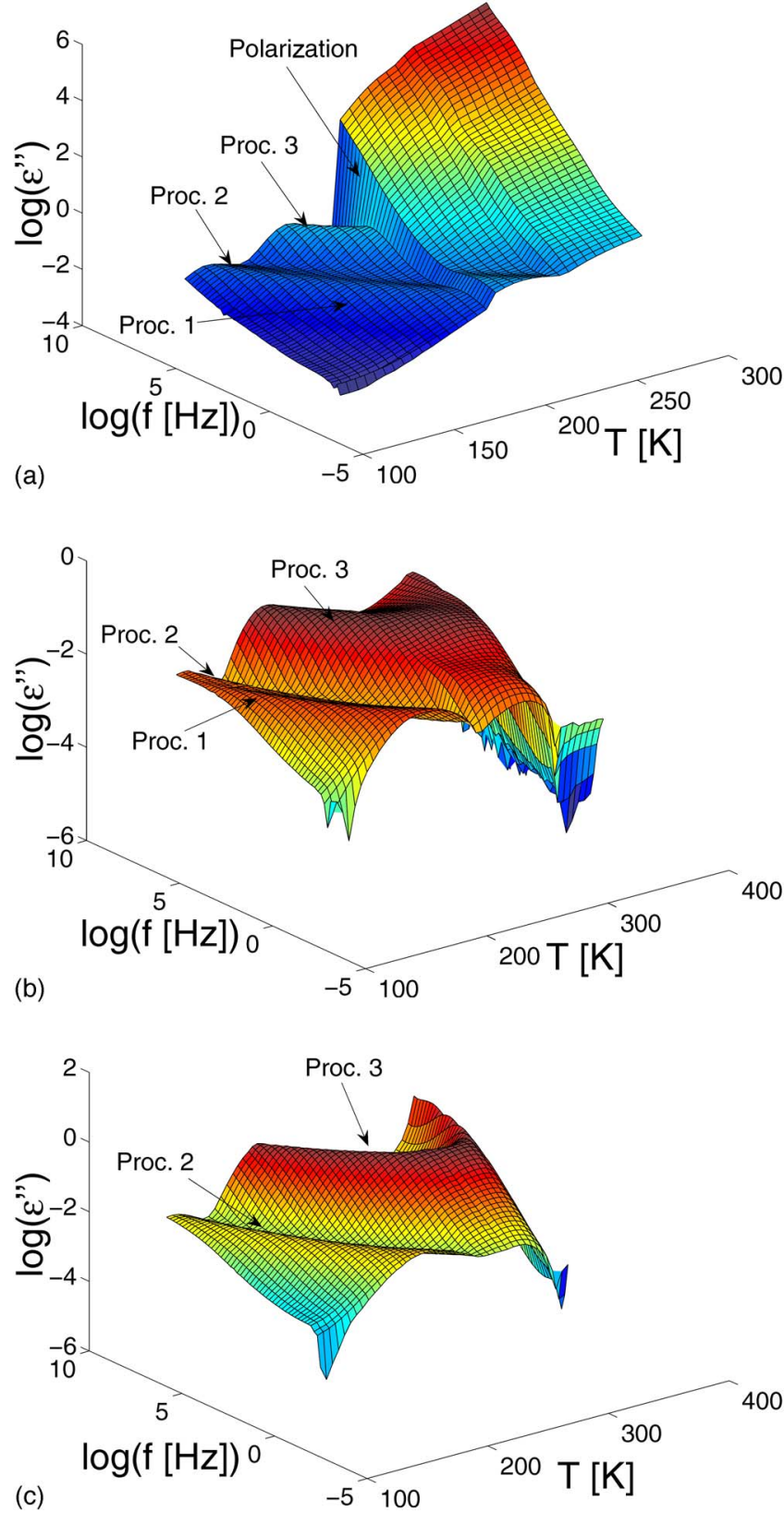

FIG. 2. (Color online) (Top) Dielectric loss of $\mathrm{C} 18$ with $\mathrm{H}=39 \%$ without a Teflon sheet. Note how electrode polarization enters between temperatures 200 and $205 \mathrm{~K}$. Note also the change in the spectrum at $240 \mathrm{~K}$, the melting point for water in $\mathrm{C} 18$ (Ref. 27). Middle: dielectric loss of $\mathrm{C} 18$ with $\mathrm{H}$ $=33 \%$ using the Teflon sheet. Process 1, 2, and 3 are all visible. (Bottom) The dielectric loss of MCM-41 C18 with $\mathrm{H}=26 \%$. The only sample where electrode polarization was completely avoided without using the Teflon sheet. The spectra clearly reveals the saddlelike temperature dependence of the high temperature/low frequency process.

rotations. For samples exposed to atmospheric conditions three different processes can be identified (depending on the hydration level). In Fig. 2 (middle) all three processes are visible for the C18 sample. The two processes at low temperatures show "normal" temperature behavior with decreasing relaxation time for increasing temperature. The high temperature process, however, first becomes faster with increasing temperature but thereafter the relaxation time reaches a minimum and show the saddlelike temperature dependence previously noticed for other systems. ${ }^{22-25}$ Unfortu- 


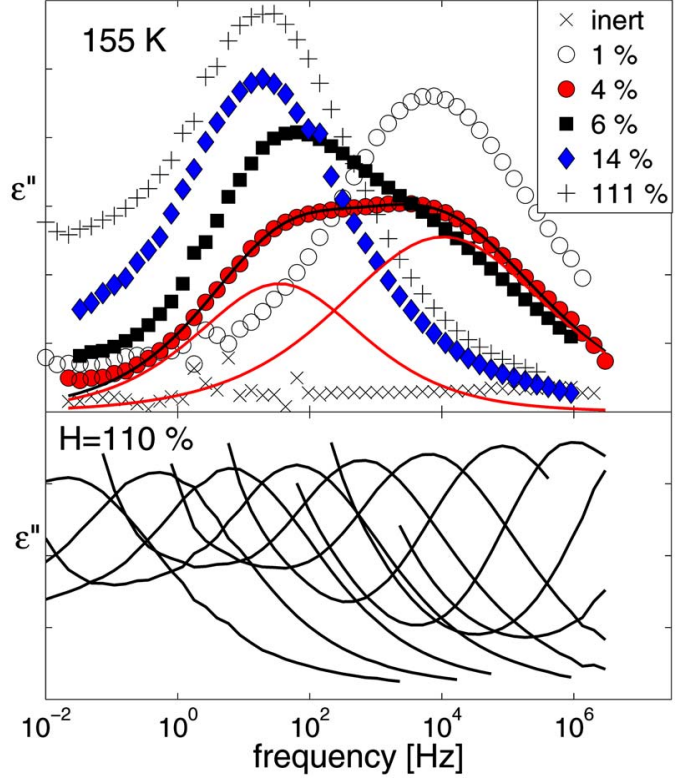

FIG. 3. (Color online) Dielectric loss of MCM-41 C10. (Top) $\epsilon^{\prime \prime}$ at $155 \mathrm{~K}$ for different hydration levels. The fit with two $\mathrm{C}-\mathrm{C}$ functions for the $\mathrm{H}$ $=4 \%$ sample is also shown (solid lines). (Bottom) $\epsilon^{\prime \prime}$ for $\mathrm{H}=110 \%$ every tenth temperature from 130 to $220 \mathrm{~K}$ from left to right.

nately, thethird process is often completely buried in a large low frequency dispersion entering the spectra above $160 \mathrm{~K}$ (Fig. 2, top). This effect is avoided by using a thin Teflon sheet between the sample and the upper electrode (Fig. 2, middle).

\section{1. $C 10(21 \AA)$}

While the inert samples showed no relaxation process the samples exposed to atmospheric conditions after evacuation showed a process entering the spectrum at $120 \mathrm{~K}$ (process 1) and shifts to higher frequencies with increasing temperature. It is satisfyingly fitted with a symmetric C-C function. Since no surface hydroxyls contribute to any relaxation (as seen from the inert samples) after evacuated at $573 \mathrm{~K}$ this process is attributed to monolayer water closely associated with the pore walls. The relaxation time is described by Eq. (1) throughout the whole temperature range (see Fig. 3).

When measuring more hydrated samples the relative strength of process I decreases on behalf of a second process (process 2) that appears roughly three decades lower in frequency. It starts to be visible for a hydration level of a few percent. The strength of this process increases rapidly with only a small increase in $\mathrm{H}$ until it totally dominates the spectra and is therefore assigned to supercooled hydration water

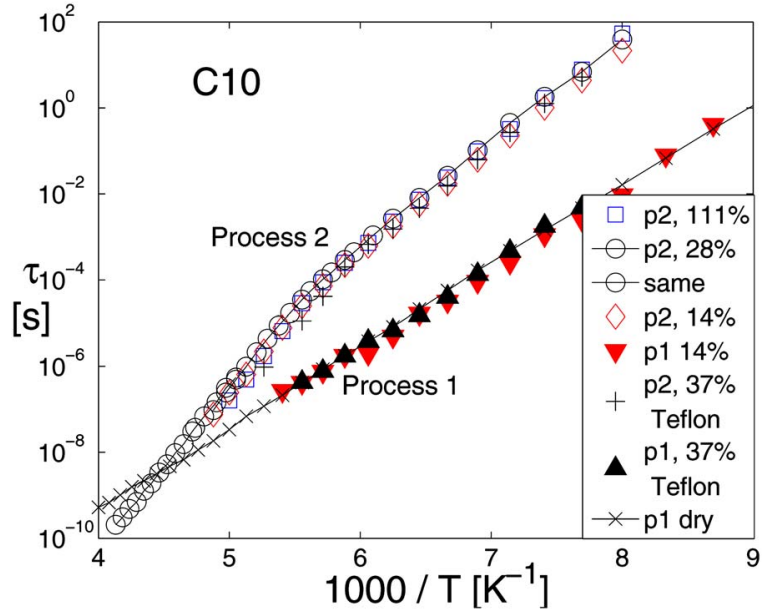

FIG. 4. (Color online) Arrhenius plot of processes 1 (filled symbols) and 2 (open symbols) obtained from the $\mathrm{C}-\mathrm{C}$ fits of the imaginary part of the spectra from $\mathrm{C} 10$ for different hydration levels. Note that neither the hydration nor the presence of the Teflon sheet affects the relaxation time.

(capillary condensed). The peak is also fitted with a $\mathrm{C}-\mathrm{C}$ function and both its shape parameter and its relaxation time agree with that of supercooled water confined in a huge number of systems. ${ }^{12,13}$ As can be seen from Fig. 3 the relaxation times of both processes is independent of hydration level and only their relative strengths are affected.

Figure 4 shows an Arrhenius plot for these processes. Again it indicates hydration independent relaxation times and shape parameters of both hydration and monolayer water. Note also that the relaxation times of both processes are almost unaffected by the presence of the Teflon sheet. The relaxation time of process 2 shows two different Arrhenius dependencies in the measured temperature range. It exhibits a kink at about $180 \mathrm{~K}$ where the activation energy increases from approximately $45 \mathrm{~kJ} / \mathrm{mol}$ to roughly $70 \mathrm{~kJ} / \mathrm{mol}$. The relaxation time extrapolates to unphysical relaxation times for high temperatures and it is therefore suggested that it is described by a non-Arrhenius (VFT) behavior at even higher temperatures. Table I shows the details of the Arrhenius fit as well as the broadening parameter of processes 1 and 2 .

For higher temperatures the polarization dispersion dominates the spectra, but by using Teflon sheet the polarization contribution is markedly reduced and a third process (process 3) is clearly visible. The peak cannot be exactly described by only one $\mathrm{H}-\mathrm{N}$ function. The peak frequency of the process can, however, be followed. It exhibits the already mentioned saddlelike temperature dependence and the average relaxation time is well described by Eq. (2). This becomes much faster with increasing hydration level.

TABLE I. Fit parameters of the Cole-Cole equation of processes 1 and 2 (monolayer and capillary condensed hydration water) and the fit to Arrhenius behavior.

\begin{tabular}{|c|c|c|c|c|c|c|c|c|c|}
\hline & \multicolumn{3}{|c|}{ Proc. 1} & \multicolumn{3}{|c|}{ Proc. $2, T \leqslant 170 \mathrm{~K}$} & \multicolumn{3}{|c|}{ Proc. $2, T \geqslant 180 \mathrm{~K}$} \\
\hline & $\alpha$ & $E_{a}[\mathrm{~kJ} / \mathrm{mol}]$ & $\tau_{0}[\mathrm{~s}]$ & $\alpha$ & $E_{a}[\mathrm{~kJ} / \mathrm{mol}]$ & $\tau_{0}[\mathrm{~s}]$ & $\alpha$ & $E_{a}[\mathrm{~kJ} / \mathrm{mol}]$ & $\tau_{0}[\mathrm{~s}]$ \\
\hline $\mathrm{C} 10$ & 0.38 & $38-40$ & $5 \times 10^{-18}$ & $0.45-0.48$ & $45-47$ & $2 \times 10^{-18}$ & $0.47-0.49$ & $67-74$ & $10^{-27}$ \\
\hline $\mathrm{C} 18$ & $0.4-0.42$ & $36-39$ & $5 \times 10^{-18}$ & $0.43-0.47$ & $43-47$ & $5 \times 10^{-18}$ & $0.42-0.46$ & $58-70$ & $10^{-24}$ \\
\hline
\end{tabular}




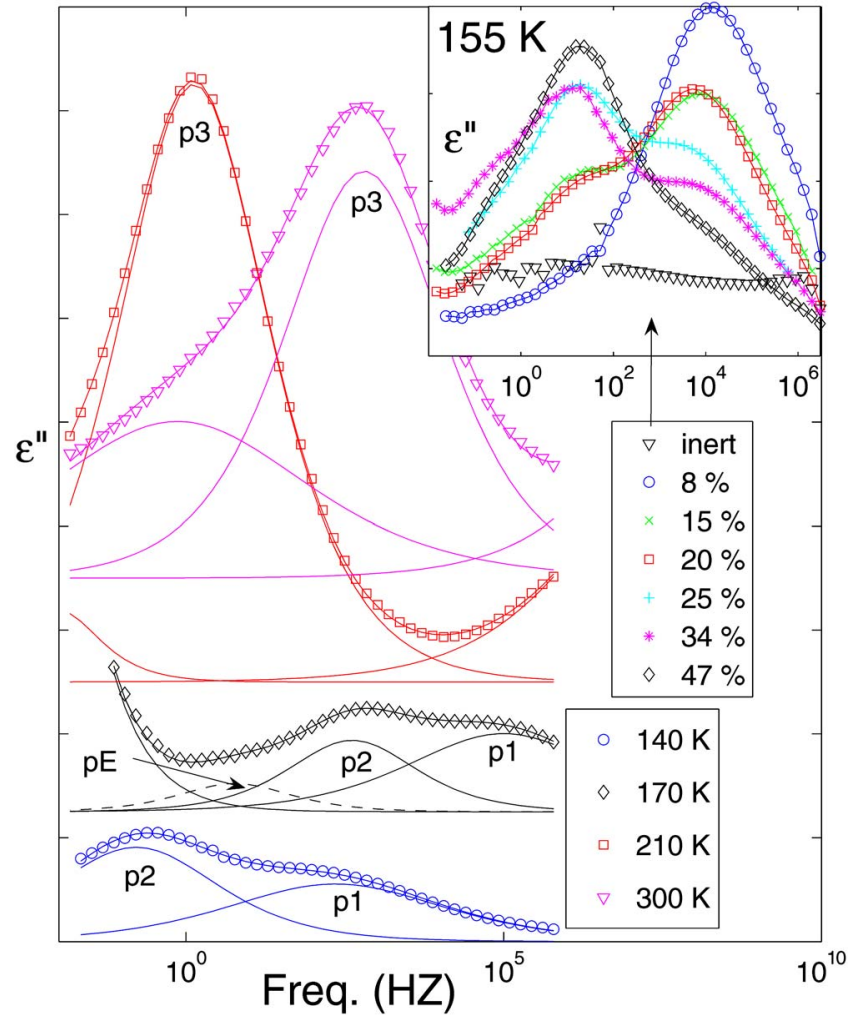

FIG. 5. (Color online) Dielectric loss of MCM-41 C18, $\mathrm{H}=34 \%$, for $T$ $=140,170,210$, and $300 \mathrm{~K}$. The fits with three $\mathrm{H}-\mathrm{N}$ functions are also indicated. The spectra are shifted to higher loss for better visibility, however, the scale is the same. The inset shows $\epsilon^{\prime \prime}$ at $155 \mathrm{~K}$ for $\mathrm{C} 18$ with different hydration levels. Note that the relaxation times of processes I and II stays virtually the same regardless of the relaxation time. Note also that the inert sample shows no relaxation process.

\section{2. $C 18(36 \AA)$}

Just as for the smaller pores the inert C18 (36 $\AA$ ) samples show no dielectric process. And again, other samples show only one relaxation for low temperatures and low $\mathrm{H}$. The process is marginally broader than process 1 for C10 and the peak is located at somewhat lower frequencies. The relaxation time exhibits an Arrhenius dependence and is naturally assigned to monolayer water as the equivalent process for the C10 sample.

With increasing $\mathrm{H}$ the second process appears, but at a slightly larger hydration level $(\mathrm{H} \simeq 15 \%)$ compared to $\mathrm{C} 10$. The peak is symmetric and just as broad as for the smaller pores. The relaxation time follows two Arrhenius dependencies which intersect at $\sim 180 \mathrm{~K}$ just as for the smaller C10 pores. The process is naturally attributed to capillary condensed hydration water within the pores. From Figs. 5 and 6 we conclude that the motions of hydration water and monolayer water are almost independent of hydration level also in these pores. It is also noted, by comparing Figs. 3 and 5 that the relaxation times of the monolayer and the capillary condensed hydration water is virtually independent on pore size.

To perfectly fit the C18 spectra an additional $\mathrm{H}-\mathrm{N}$ function is needed for frequencies about two decades lower than process II. This function is always about one third the strength of process II and is never resolved as a peak. It is only needed to fit the spectra when ice is present. This extra

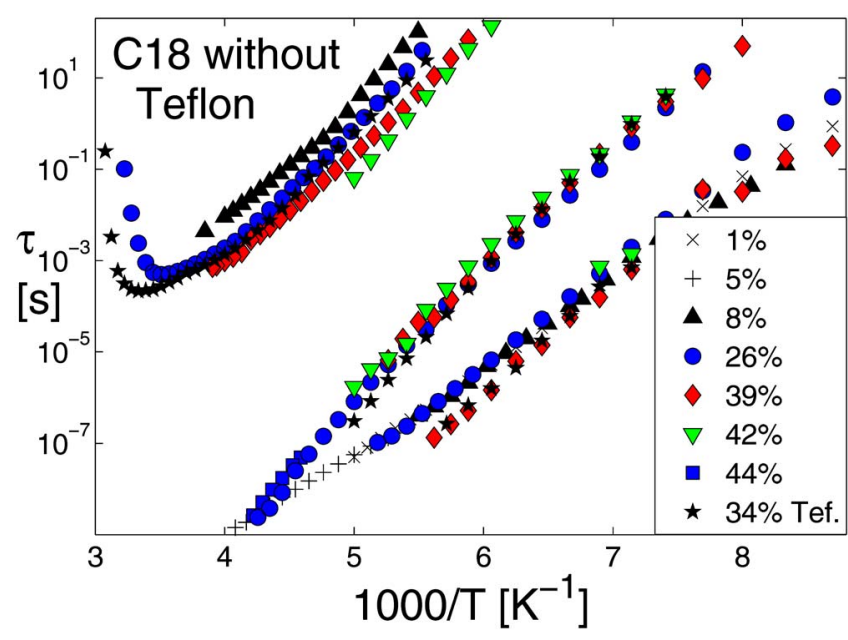

FIG. 6. (Color online) Arrhenius plot of $\tau$ from fits of Eq. (3) to the dielectric loss spectra of $\mathrm{C} 18$ for different $\mathrm{H}$. Note that the relaxation times seem little affected by the presence of the Teflon sheet.

process is labeled $\mathrm{pE}$ in Fig. 5. We conclude that this process is related to the formation of ice clusters and omit it from the discussion hereafter.

For the $\mathrm{C} 18$ samples the polarization contribution can be avoided to higher temperatures until it suddenly appears at $T=200-260 \mathrm{~K}$. For the polarization free spectra a third process of considerable strength appears at $T \simeq 160-190 \mathrm{~K}$. Only for one sample a polarization free spectra were obtained to $350 \mathrm{~K}$ where measurements stopped. The full shape and relaxation time of process 3 could then be followed. The relaxation time show the atypical saddlelike temperature dependence. In contrast to process 3 measured for $\mathrm{C} 10$ with Teflon this peak is perfectly fitted with a single $\mathrm{H}-\mathrm{N}$ function. The shape of the peak is very narrow with $\alpha \simeq 0.8$ and $\beta \simeq 0.7$. The dielectric strength is almost constant throughout the temperature interval $(\Delta \epsilon \simeq 4)$.

When applying the Teflon cap to the $\mathrm{C} 18$ samples the peak of process 3 is slightly distorted. Once again it requires two $\mathrm{H}-\mathrm{N}$ functions to fit the data satisfyingly. One function dominates, however, and it very much resembles the one found without Teflon. From Fig. 7 it is evident that the peak of this function seems to describe the same behavior as pro-

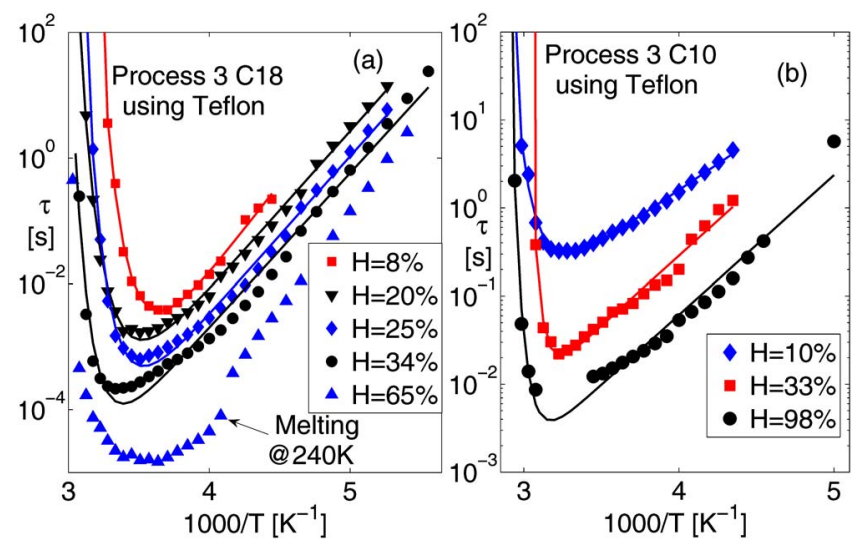

FIG. 7. (Color online) Arrhenius plot of $\tau$, the relaxation time of process 3 for (a) C18 and (b) C10. All samples were measured using the Teflon sheet. The solid lines are fits to the previously suggested model [Eq. (2)]. 
cess 3 measured without Teflon. The relaxation times for different $\mathrm{H}$ levels are shown in Fig. 7 together with fits to Eq. (2). For large $\mathrm{H}$ it is difficult to fit the data because of an abrupt change in the dynamics at $240 \mathrm{~K}$ due to the melting of ice in these pores. One example of this is shown in Fig. 7. Where no substantial crystallization occurs the data are well fitted by the model. There is, however, a lack of correlation between the fit parameters in Eq. (2) and the pore sizes of all studies.

\section{DISCUSSION}

\section{A. Crystallization in the pores}

The DSC measurements show no ice formation in the C10 pores. Some crystallization of surface water (on the outside of the grains) is sometimes detected, but the melting enthalpy never corresponds to more than about $4 \%$ of the total uptake of water. The extensive data of Ref. 27 shows that water in pores of this size should, if possible, crystallize at well below $200 \mathrm{~K}$, but in this region no peak appears. Instead, the occasional endothermic peak on upscan is found just below $273 \mathrm{~K}$, characteristic of melting surface water on the outside of the pores.

The melting in $\mathrm{C} 18$ pores shows that not all water crystallizes though ice formation becomes substantial at large hydration levels. Thus, some regions of amorphous capillary condensed hydration water remains.

The enthalpy change due to melting seems only weakly influenced by the cooling rate. For samples quenched in liquid nitrogen before upscan the enthalpy change is only decreased by $4 \%$ compared to the same sample cooled by $10 \mathrm{~K} / \mathrm{min}$. This is understandable considering the difficulties of vitrifying bulk water. If the environment allows crystallization of the water it will crystallize even for very high cooling rates.

\section{B. The dynamics of amorphous water at low temperatures}

The attribution of process 2 in the $\mathrm{C} 10$ pores to supercooled water is definite due to the already mentioned similarities with water in many different confining systems ${ }^{12,13}$ and the fact that it is totally dominating the spectrum for intermediate and large levels of hydration in $\mathrm{C} 10$ where no crystallization occurs. It seems therefore realistic to attribute the equivalent process found for the $\mathrm{C} 18$ pores to the local relaxation of amorphous water since we detect only partial crystallization under these circumstances.

Process 1 for both pore sizes is attributed to monolayer of water since the inert sample showed that not enough hydroxyl groups remained after the evacuation treatment. Unfortunately the experimental frequency window limits us to investigate processes 1 and 2 only at temperatures below $240 \mathrm{~K}$, the melting temperature of ice in C18.

This study does not support the hydration dependence of the relaxation time found for water in $32 \AA$ MCM-41, Ref. 19. Our findings indicate rather hydration independent relaxation time and shape parameters for process 2. Possibly, the hydration dependence of the electrode polarization is responsible for apparent shifts of the peaks in Ref. 19. Furthermore, the presence of ice in the pores, as shown in Ref. 27, is definitely in conflict with the assumption that only amorphous water and $\mathrm{OH}$ groups are present.

\section{The effect of the Teflon sheet}

The presence of the Teflon sheet is not affecting the relaxation time of process 1 or 2 . Neither does it seem to significantly affect the relaxation time of process 3 . Nevertheless, it does have an effect on the shape of the peak. This could be due to a Maxwell-Wagner (M-W) process ${ }^{44}$ from the interface between the regions of the hydrated MCM41 and the Teflon sheet. Introducing a two-layer model ${ }^{33}$ of the system and assuming that $\sigma_{\text {Teflon }} \ll \sigma_{\text {MCM-41 }}$ and $\epsilon_{\text {Teflon }}^{\prime}$ $\ll \epsilon_{\text {MCM-41 }}^{\prime}$, where $\sigma$ is the dc conductivity, one obtain a rough estimation of the relaxation time of a $\mathrm{M}-\mathrm{W}$ process: $\tau_{\mathrm{M}-\mathrm{W}} \simeq \epsilon_{0} \epsilon^{\prime} / \sigma$ where the values correspond to the pure sample. The dc conductivity of hydrated MCM-41 is not available from the measurements but it can be estimated to be less than $10^{-12} \mathrm{~S} / \mathrm{m}$ at $300 \mathrm{~K}$ and the relaxation time of any $\mathrm{M}-\mathrm{W}$ peak would then be no smaller than $10 \mathrm{~s}$. It is thus possible that the change in the shape of the peak when the Teflon is introduced is due to a submerged M-W peak induced by the sample/Teflon interface.

\section{Apparent fragile-to-strong transitions}

The discrepancy of the fragility of water on either side of the no mans land suggests the existence of a FST somewhere between $150-235 \mathrm{~K}$ of bulk water. The fact that thermodynamical arguments ${ }^{34}$ predict a peak at $220 \mathrm{~K}$ for the heat capacity of water also suggests a change in the activation energy according to the Adam-Gibbs equation: $\tau$ $=\tau_{0} \exp \left(C / T S_{c}\right)$, where $S_{c}$ is the configurational entropy. ${ }^{35}$ There is no doubt that a crossover of the $\mathrm{H}$ diffusivity occurs around $220 \mathrm{~K}$ in QENS, ${ }^{3} \mathrm{NMR},{ }^{5}$ and also neutron spin echo experiments. ${ }^{36}$ However, the abrupt behavior of the transition seems very pronounced and the low temperature relaxation time extrapolates to an unrealistic $T_{g}$. It is therefore unlikely that the diffusivity in these studies is coupled to the structural relaxation. This was recently suggested for water in different confinements $^{13}$ and also experimentally verified for hydrated lysozyme. 37

This study shows that dielectric relaxation does not participate in a FST up to $240 \mathrm{~K}$. A change in fragility should be visible in dielectric relaxation measurements which have been proven to probe reorientational motions better than most other techniques for polar liquids. An inspection of the $\mathrm{H}$ diffusivity in ice ${ }^{38}$ shows that ice exhibits a very high diffusion rate of water molecules. In fact, the diffusivity of confined amorphous water ${ }^{5}$ is closer to that of ice than that of supercooled bulk water. ${ }^{39}$ Since no structural relaxation occurs in ice this suggests that: (i) A breakdown of the StokesEinstein relation $\left(D \propto \tau^{-1}\right)$ occurs and the crossover cannot be related to structural relaxation and, consequently, viscosity or the fragility. (ii) One should be careful when relating properties of confined water to the properties of the bulk state.

Note that even though the dielectric relaxation does not exhibit a FST we cannot rule out the possible existence of a critical point at $p_{c}=1600$ bars and $T_{c}=200 \mathrm{~K}$ as suggested 
in, e.g., Ref. 4. More discontinuous changes of the structure, reorientational motions, or density might occur for such temperatures at elevated pressure. It would therefore be interesting to see a high pressure dielectric and calorimetric study of these systems.

One might suggest that the change in the temperature dependence of the dielectric relaxation time detected at $180 \mathrm{~K}$ for process II could correspond to a FST. The physical (mathematical) origin of the apparent change in the dielectric relaxation is discussed in a previous paper. ${ }^{14}$ It is explained by Williams ansatz ${ }^{40}$ when a local process merges with a more cooperative motion, even though the strength of the cooperative motion is vanishingly small ${ }^{41}$ due to the confinement. The finite volume is thus insufficient for forming the H-bonded network necessary for extended cooperative motions. For temperatures below $180 \mathrm{~K}$ the main process of confined water is therefore considered to be of local character in agreement with the nonvisible calorimetric glass transition and the dynamical behavior of hydrated proteins at these temperatures. ${ }^{13}$

\section{E. The nature of the high temperature process (process 3)}

We now focus on the origin of process 3 with the saddlelike temperature dependence. As previously mentioned such processes have been reported four times before in the literature. $^{22-25}$ They have all been attributed to inner surface water closely associated with the pore walls and explained by the model detailed in Ref. 23 . The slowing down for high temperatures is said not to originate in loss of water even though the systems showing this behavior have great loss of water for $T \sim$ room temperature. The reason is that the maximum loss appears some $50 \mathrm{~K}$ above the saddlepoint and that the dielectric strength of the process increases slowly with temperature throughout the temperature interval. In the following we investigate the equivalent process of this system and relate it to the previous explanations.

In Fig. 8 the relaxation time of process 3 from a sample of $\mathrm{C} 10$ with $\mathrm{H}=29 \%$ is shown. The sample is cooled using the cryostat of the instrument. Measurements are performed every $5^{\circ}$ on heating. Repeated measurements are done at $T$ $=270 \mathrm{~K}$ after measurements at 295, 305, and $320 \mathrm{~K}$. The process appears with the typical activation energy for low temperatures. At $295 \mathrm{~K}$ a tendency to slow down is noticed. Remeasuring at $270 \mathrm{~K}$ then reveals that the relaxation time is longer than when first measured at this temperature. Increasing the temperature to $300 \mathrm{~K}$ shows that the saddlelike process continues as expected. After visiting $305 \mathrm{~K}$ the relaxation time remeasured at $270 \mathrm{~K}$ is even longer and after measuring at $320 \mathrm{~K}$ the process at $270 \mathrm{~K}$ is almost one decade slower than the first time measured. At the same time the dielectric strength of the process at $270 \mathrm{~K}$ is reduced after excursions to higher temperatures even though this is maintained at the higher temperatures. The decrease is however only $21 \%$.

Doing similar measurements at $170 \mathrm{~K}$ after measuring every $5^{\circ}$ up to $340 \mathrm{~K}$ reveals that the dielectric strength of

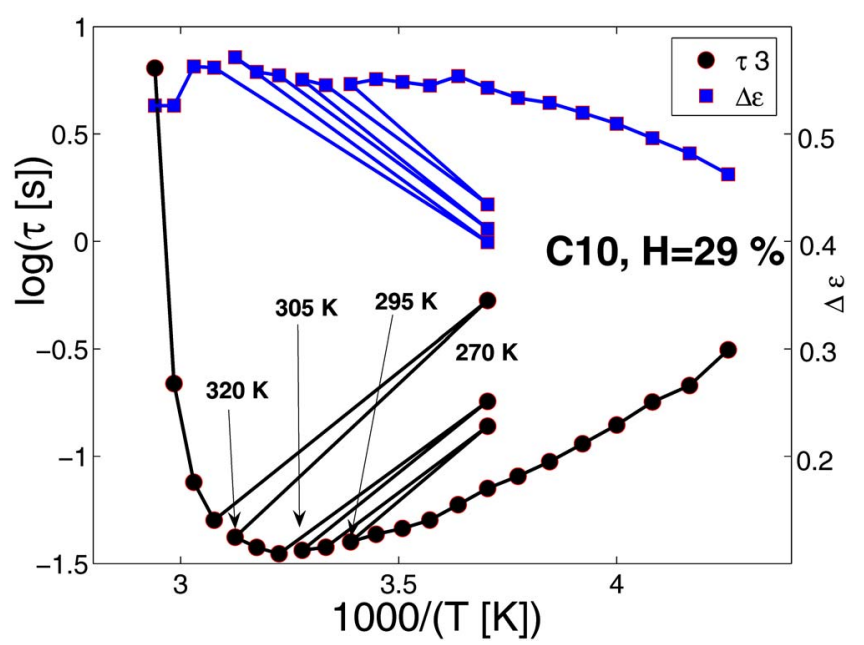

FIG. 8. (Color online) MCM-41 C10, $\mathrm{H}=39 \%$ measured each $5 \mathrm{~K}$ from 235 to $350 \mathrm{~K}$. The spectrum at $270 \mathrm{~K}$ is remeasured after probing at 295 , 305 , and $320 \mathrm{~K}$. The chronological path can be followed from right to left by the solid line. The figure shows the average relaxation time $(\tau)$ and dielectric strength $(\Delta \epsilon)$ of process 3 , obtained from the $\mathrm{H}-\mathrm{N}$ fit of the spectra. Note the drastic slowing down of the dynamics at $270 \mathrm{~K}$ when the hydration level decreases (due to measurements at higher temperatures).

process 2 , the hydration water, is decreased by almost $90 \%$. Measurements at higher temperatures thus causes a substantial loss of hydration water.

From the result of Fig. 8 we argue that the drastic slowing down is due to loss of capillary condensed hydration water. What is then the origin of the process? It could be relaxation of a third species of confined water within our system or a MW process due to the heterogeneity of the sample. The different dc conductivity in the pores and the silica matrix can cause charge accumulation which relaxes just like a rotating dipole. It is clarified that the monolayer and hydration water relaxes according to processes 1 and 2 and that IR measurements ${ }^{27}$ cannot specify a third species of water.

Even though the data is well described by Eq. (2) the lack of correlations between the fit parameters and the pore sizes are suspicious. Therefore we investigate the possibility of a $\mathrm{M}-\mathrm{W}$ process being the origin of this process. The theory for the $\mathrm{M}-\mathrm{W}$ effect has been treated for a number of different geometries of the heterogeneity. ${ }^{33}$ Most of them result in a Debye-like dispersion. In reality some broadening of the process should occur due to variations of the sample geometry. In fact, the narrow shape of process 3 in this study is very unusual for confined water which favors the attribution of a M-W process.

No theory for dispersed domains of aligned cylinders has been reported in the literature. We therefore use a model with a simplified geometry, dispersed spheres in silica treated in Ref. 33. The system is defined by spheres of water, with dielectric constant $\epsilon_{w}$ and conductivity $\sigma_{w}$, dispersed in a much larger sphere of $\mathrm{SiO}_{2}$ (dielectric constant $\epsilon_{s}$ and conductivity $\sigma_{w}$ ). By calculating the admittance of such a system the contribution to the complex permittivity of a $\mathrm{M}-\mathrm{W}$ process is given by the Debye equation 


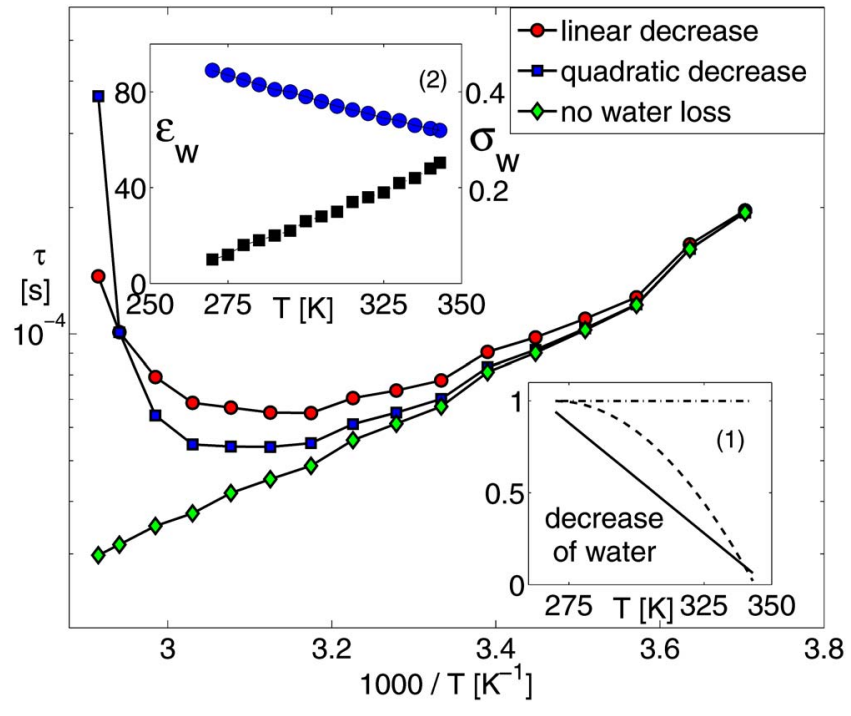

FIG. 9. (Color online) Calculation of the peak maximum of the MaxwellWagner peak in a low conducting material with dispersed high conductivity spheres according to Eq. (6). Circles and squares represent calculated peak maximum (M-W relaxation time) where conductivity and dielectric constant of water is scaled by the linear and quadratic function of inset 1 , respectively. Diamonds represent the scenario of no loss of water. In this case the conductivity and dielectric constant varies only with temperature as the bulk values, shown in the upper inset. The minimum relaxation time occurs for $T=315$ and $320 \mathrm{~K}$ for the linear and quadratic model, respectively. This is within $10 \%$ of the experimental result. (Inset 1) The linear and quadratic functions used to model the loss of water. (Inset 2) Typical bulk values for the dielectric constant and conductivity of the water in this study.

$$
\epsilon_{\mathrm{MW}}^{*}=\epsilon_{\infty}+\frac{\epsilon_{\mathrm{stat}}-\epsilon_{\infty}}{1+i \omega \tau_{\mathrm{MW}}} .
$$

The expressions for $\epsilon_{\text {stat }}, \epsilon_{\infty}$, and $\tau_{\mathrm{MW}}$ are relatively simple for $\sigma_{s} \ll \sigma_{w}$,

$$
\begin{aligned}
& \epsilon_{\text {stat }}=\epsilon_{s} \frac{1+2 v_{w}}{v_{s}}, \quad \epsilon_{\infty}=\epsilon_{s} \frac{2 \epsilon_{s}+\epsilon_{w}+2 v_{w}\left(\epsilon_{w}-\epsilon_{s}\right)}{2 \epsilon_{s}+\epsilon_{w}-v_{w}\left(\epsilon_{w}-\epsilon_{s}\right)}, \\
& \tau_{\mathrm{MW}}=\epsilon_{0} \frac{2 \epsilon^{\prime}+\epsilon^{\prime}-v_{w}\left(\epsilon_{w}^{\prime}-\epsilon_{s}^{\prime}\right)}{v_{s} \sigma_{w}},
\end{aligned}
$$

where $v_{w}$ and $v_{s}=1-v_{w}$ are the volume fractions of water and the silica matrix, respectively (set to 0.5 each in this example).

The temperature dependent conductivity and dielectric constant of water in the range of $T=270-340 \mathrm{~K}$ are taken from Ref. 42 and displayed in the upper inset of Fig. 9. The dielectric constant of $\mathrm{SiO}_{2}$ is set to $\epsilon_{s}^{\prime}=3.8 .^{43}$ To incorporate the loss of water we assume that the dielectric constant and conductivity within the pores decreases with decreasing water content. Since the dielectric constant is proportional to the number of relaxing dipoles we assume a linear relationship between $\epsilon_{w}$ and water content. How the conductivity decreases on decreasing water content is difficult to predict but in this rough estimation a linear relation is assumed also for $\sigma_{w}$. Using two different functions for the loss of water, one linear and one quadratic, that both have full water content at $165 \mathrm{~K}$ and only $10 \%$ remaining at $340 \mathrm{~K}$, we can plot the temperature dependence of the peak maximum of the $\mathrm{M}-\mathrm{W}$ process. The result is shown in Fig. 9.
The figure shows almost Arrhenius dependence of the process if no water escapes the pores. It is clear that the deviation from Arrhenius dependence for high temperatures occurs when the water content decreases. The slowing down is very drastic with what seems to be described by a double exponential as in Eq. (2). The effect is more pronounced with the quadratic decrease of water compared to the linear. The relaxation time of the processes in Fig. 9 coincide very well with the process for the $\mathrm{C} 18$ pores at large hydration levels. Note also that the saddle point occurs well below the maximum loss rate of water in the quadratic model. This contradicts the arguments that loss of water is not responsible for the slowing down. ${ }^{22}$ The dielectric strength of the process is about constant throughout the temperature interval with $\Delta \epsilon$ $\sim 3.5$, which is the same order of magnitude as experimental values (the exact strength in experiments cannot be determined due to uncertainty in sample thickness).

The results of Fig. 9 are not intended to give an exact description of the $\mathrm{M}-\mathrm{W}$ effect in these materials. Rather, it shows that even simple models of the M-W effect combined with intuitive arguments produces a M-W peak which exhibits the same temperature dependence, same relaxation times and similar dielectric strength as the one measured in this study and shown for other systems.

\section{CONCLUSIONS}

Our dielectric study of water confined in MCM-41 materials with pore sizes 21 and $36 \AA$ reveals the dynamics of supercooled capillary condensed hydration water and unfreezable monolayer water. The results show that the motions of hydration water and monolayer water are virtually independent of the hydration level and pore size, in contrast to previous studies. ${ }^{19}$ The dielectric relaxation of the hydration water does not exhibit any fragile-to-strong transition for temperatures up to $240 \mathrm{~K}$. The calorimetric measurements reveal that only partial crystallization of the capillary condensed water occurs in the larger pores. No crystallization occurred for the smaller pores.

A large dispersion entering the spectra at high temperatures shows a saddlelike temperature dependence of its relaxation time. It is shown that the drastic slowing down of this process at high temperatures is due to loss of water from the matrix. The characteristic relaxation time of this process shows a dependence on both pore size and hydration level. Similar behavior can be expected for Maxwell-Wagner processes when the system loses water. In conclusion we therefore attribute this process to relaxation of accumulated charges within the pores. We suggest that this is also the case for relaxation showing saddlelike temperature dependence for water in other porous materials, in contradiction to previous explanations. $^{22}$

\section{ACKNOWLEDGMENTS}

This work was financially supported by the Swedish Research Council and the Swedish Foundation for Strategic Research. J.S. is a Royal Swedish Academy of Sciences Research fellow supported by a grant from the Knut and Alice Wallenberg Foundation. 
${ }^{1}$ P. G. Debenedetti, J. Phys.: Condens. Matter 15, R1669 (2003).

${ }^{2}$ K. Ito, C. T. Moynihan, and C. A. Angell, Nature (London) 398, 492 (1999).

${ }^{3}$ A. Faraone, L. Liu, C.-Y. Mou, C.-W. Yen, and S.-H. Chen, J. Chem. Phys. 121, 10843 (2004).

${ }^{4}$ L. Liu, S.-H. Chen, A. Faraone, C.-W. Yen, and C.-Y. Mou, Phys. Rev. Lett. 95, 117802 (2005).

${ }^{5}$ F. Mallamace, M. Broccio, C. Corsaro, A. Faraone, U. Wanderlingh, L. Liu, C.-Y. Mou, and S.-H. Chen, Phys. Rev. Lett. 95, 117802 (2005).

${ }^{6}$ E. Mamontov, J. Chem. Phys. 123, 171101 (2005).

${ }^{7}$ S. H. Chen, L. Liu, E. Fratini, A. Faraone, and E. Mamontov, Proc. Natl. Acad. Sci. U.S.A. 103, 9012 (2006).

${ }^{8}$ L. M. Xu, P. Kumar, S. V. Buldyrev, S. H. Chen, P. H. Poole, F. Sciortino, and H. E. Stanley, Proc. Natl. Acad. Sci. U.S.A. 102, 16558 (2005).

${ }^{9}$ J. Swenson, Phys. Rev. Lett. 97, 189801 (2006).

${ }^{10}$ S. Cerveny, J. Colmenero, and A. Alegria, Phys. Rev. Lett. 97, 189802 (2006).

${ }^{11}$ S. H. Chen, L. Liu, and A. Faraone, Phys. Rev. Lett. 97, 189803 (2006).

${ }^{12}$ S. Cerveny, G. A. Schwartz, R. Bergman, and J. Swenson, Phys. Rev. Lett. 93, 245702 (2004).

${ }^{13}$ J. Swenson, H. Jansson, and R. Bergman, Phys. Rev. Lett. 96, 247802 (2006).

${ }^{14}$ J. Hedström, J. Swenson, R. Bergman, and S. Kittaka, Eur. Phys. J. Spec. Top. 141, 53 (2007).

${ }^{15}$ S. Cerveny, J. Colmenero, and A. Alegria, J. Non-Cryst. Solids 353, 4523 (2007)

${ }^{16}$ S. Capaccioli, K. L. Ngai, and N. Shinyashiki, J. Phys. Chem. B 111, 8197 (2007).

${ }^{17}$ J. Y. Ying, C. P. Mehnert, and M. S. Wong, Angew. Chem., Int. Ed. 38, 56 (1999).

${ }^{18}$ M. Kinka, J. Banys, J. Macutkevic, A. Poppl, W. Bohlmann, V. Umamaheswari, M. Hartmann, and G. Volkel, Phys. Status Solidi B 242, R100 (2005).

${ }^{19}$ A. Spanoudaki, B. Albela, L. Bonneviot, and M. Peyrard, Eur. Phys. J. E 17, 21 (2005).

${ }^{20}$ J. Banys, M. Kinka, J. Macutkevic, G. Volkel, W. Bohlmann, V. Umamaheswari, M. Hartmann, and A. Poppl, J. Phys.: Condens. Matter 17, 2843 (2005).

${ }^{21}$ H. Jansson and J. Swenson, Eur. Phys. J. E 12, S51 (2003).
${ }^{22}$ L. Frunza, H. Kosslick, I. Pitsch, S. Frunza, and A. Schönhals, J. Phys. Chem. B 109, 9154 (2005).

${ }^{23}$ Y. Ryabov, A. Gutina, V. Arkhipov, and Y. Feldman, J. Phys. Chem. B 105, 1845 (2001).

${ }^{24}$ A. Gutina, E. Axelrod, A. Puzenko, E. Rysiakiewicz-Pasek, N. Kozlovich, and Y. Feldman, J. Non-Cryst. Solids 302, 235 (1998).

${ }^{25}$ L. Frunza, H. Kosslick, S. Frunza, and A. Schönhals, J. Phys. Chem. B 106, 9191 (2002).

${ }^{26}$ T. Mori, Y. Kuroda, Y. Yoshikawa, M. Nagao, and S. Kittaka, Langmuir 18, 1595 (2002).

${ }^{27}$ S. Kittaka, S. Ishimaru, M. Kuranishi, T. Matsuda, and T. Yamaguchi, Phys. Chem. Chem. Phys. 8, 3223 (2006).

${ }^{28}$ S. Takahara, M. Nakano, S. Kittaka, Y. Kuroda, T. Mori, H. Hamano, and T. Yamaguchi, J. Phys. Chem. B 103, 5814 (1999).

${ }^{29}$ S. Takahara, N. Sumiyama, S. Kittaka, T. Yamaguchi, and M. BellissentFunel, J. Phys. Chem. B 109, 11231 (2005).

${ }^{30}$ P. Smirnov, T. Yamaguchi, S. Kittaka, S. Takahara, and Y. Kuroda, J. Phys. Chem. B 104, 5498 (2000).

${ }^{31}$ C. A. Angell, J. Shuppert, and J. C. Tucker, J. Phys. Chem. 77, 3092 (1973).

${ }^{32}$ P. Debye, Polare Molekin (Hirzel, Leipzig, 1929).

${ }^{33}$ L. K. H. van Beek, Prog. Dielectr. 7, 69 (1969).

${ }^{34}$ F. W. Starr, C. A. Angell, and H. E. Stanley, Physica A 323, 51 (2003).

${ }^{35}$ G. Adam and J. H. Gibbs, J. Chem. Phys. 43, 139 (1965).

${ }^{36} \mathrm{~T}$. Yamaguchi (unpublished).

${ }^{37}$ S. Pawlus, S. Khodadadi, and A. Sokolov, Phys. Rev. Lett. 100, 108103 (2008).

${ }^{38}$ L. Onsager and L. K. Runnels, J. Chem. Phys. 50, 1089 (1969).

${ }^{39}$ K. T. Gillen, D. C. Douglass, and M. J. R. Hoch, J. Chem. Phys. 57, 5117 (1972).

${ }^{40}$ G. Williams, Adv. Polym. Sci. 33, 60 (1979).

${ }^{41}$ R. Bergman and C. Svanberg, Phys. Rev. E 72, 043501 (2005).

${ }^{42}$ R. C. Weast, CRC Handbook of Chemistry and Physics, 87th ed. (CRC, London, 2006).

${ }^{43}$ D. Hollowayt, The Physical Properties of Glass, The Wykeham Science Series (Wykeham, London, 1973).

${ }^{44} \mathrm{~A}$ polarization arising in heterogeneous samples where charges accumulate at the interfaces between regions of different permittivity or conductivity. See, i.e., Ref. 33. 